\title{
First investigation of electrochemical behavior and Detection of 2-O-( $\beta$-D-glucopyranosyl) ascorbic acid
}

Xin Shi ${ }^{1}$, Fengfeng Zhang ${ }^{1}$, Xia Liu ${ }^{1}$, Yuhong Zheng ${ }^{2, *}$, Li Fu $^{3, *}$, Haobing Shi ${ }^{3}$, Fang Wang ${ }^{1}$, and Zenglai $X u^{2}$

\author{
${ }^{1}$ Ningxia Institute of Quality Standards and Testing Technology for Agricultural Products, Yinchuan \\ 750002, PR. China \\ ${ }^{2}$ Institute of Botany, Jiangsu Province and Chinese Academy of Sciences, Nanjing Botanical Garden, \\ Mem. Sun Yat-Sen, Nanjing 210014, PR. China \\ ${ }^{3}$ Key Laboratory of Novel Materials for Sensor of Zhejiang Province, College of Materials and \\ Environmental Engineering, Hangzhou Dianzi University, Hangzhou, 310018, PR. China \\ *E-mail: zhengyuhong@ cnbg.net, fuli@hdu.edu.cn
}

Received: 6 July 2021 / Accepted: 29 August 2021 / Published: 10 October 2021

In this work, the electrochemical behavior of 2-O-( $\beta$-D-glucopyranosyl) ascorbic acid, a vitamin $\mathrm{C}$ analogue, was reported, with glassy carbon electrode as working electrode. It is the first report regarding the study of electrochemical property of 2-O-( $\beta$-D-glucopyranosyl) ascorbic acid. In addition, an analytical method for 2-O-( $\beta$-D-glucopyranosyl) ascorbic acid detection based on its electrochemical oxidation signal was established. In the range of $100 \mathrm{nM}$ to $300 \mu \mathrm{M}$, the proposed electrochemical method was allowed for linear detection. The detection limit was calculated as $30 \mathrm{nM}$ based on $\mathrm{S} / \mathrm{N}=3$. This result was more sensitive than the two HPLC-based analytical protocols that had been reported.

Keywords: Lycium barbarum; Electrochemical behavior; Chemical component; Chinese traditional medicine; Electrochemical oxidation

\section{FULL TEXT}

(C) 2021 The Authors. Published by ESG (www.electrochemsci.org). This article is an open access article distributed under the terms and conditions of the Creative Commons Attribution license (http://creativecommons.org/licenses/by/4.0/). 